\title{
Prevalência de perda de inserção periodontal Em adolescentes de 12 a 16 anos em uma Escola pública de porto alegre*
}

\author{
Prevalence of periodontal attachment loss \\ In 12 to 16-year-old adolescents in a \\ Public school in porto alegre
}

\author{
${ }^{* *}$ Carina Maciel da Silva \\ ${ }^{* * *}$ Cassiano Kuchenbecker Rösing
}

\begin{abstract}
RESUMO
O objetivo do estudo foi verificar a prevalência de perda de inserçāo (PI) em adolescentes de 12 a 16 anos de idade de uma escola pública de Porto Alegre. A prevalência de PI foi constatada através de sondagem periodontal nos ângulos mésio-vestibulares, disto-vestibulares, e no centro das faces vestibulares e linguais/palatinas dos incisivos e primeiros molares permanentes. O exame foi realizado somente por um examinador treinado, dispondo de sonda periodontal tipo Willians, espelho bucal e iluminação padrão. O nível de inserção periodontal foi definido como a distânica em milímetros do limite amelo-cementário à porção mais apical sondável, próxima ao fundo do sulco/bolsa. Para cada sítio duas medidas foram feitas, a distância da margem gengival ao limite amelo-cementário, e a distância da margem gengival ao fundo do sulco/bolsa. O nível de inserção para um dado sítio foi. então, calculado subtraindo-se o primeiro do segundo ou, no caso de recessão gengival, somando-se os dois. Considerou-se PI quando esta mensuraçāo excedeu $2 \mathrm{~mm}$ em faces livres e $3 \mathrm{~mm}$ em faces proximais. Foram examinados 3048 sítios, sendo encontrada uma prevalência de $4.82 \%$ de PI, deste valor $36.77 \%$ com presença de sangramento e $61.22 \%$ com ausência. A face vestibular mostrou-se como o sítio com a maior prevalência de PI.
\end{abstract}

\section{UNITERMOS}

inserção periodontal; epidemiologia; adolescentes.

\section{INTRODUÇÃO}

Infecção e inflamação constituem os marcos fundamentais da doença periodontal. A reação inflamatória é visível, microscópica e clinicamente, no periodonto afetado e representa a reação do hospedeiro à microbiota da placa e seus produtos. Entretanto, a reação inflamatória pode não ser de todo benéfica porque é também passível de causar dano às células e estruturas vizinhas do tecido conjuntivo, incluindo o osso alveolar ${ }^{14}$.

Dentre as afecções do periodonto esta compreende um grupo de doenças periodontais que afetam crianças e jovens adultos, sendo caracterizada por ter rápida e progressiva destruição $\mathrm{da}$ inserção periodontal ${ }^{4}$. Diversos autores têm dividido estas afecçōes em periodontite pré-puberal e periodontite juvenil ${ }^{2,13,26}$ conforme a idade do indivíduo na ocasiāo do diagnóstico.

Dentro da periodontite juvenil, há ainda uma subdivisão em duas formas de como esta se apresenta: localizada e generalizada. A periodontite juvenil localizada envolveria somente primeiros molares e incisivos permanentes ${ }^{6}$. Entretanto, há autores que acreditam que a forma generalizada seria uma evolução da localizada, pois aqueles são os primeiros dentes permanentes a erupcionarem. BROWN et al em 1996 publicaram um estudo longitudinal no qual acompanharam a perda de inserção periodontal em um grupo de adolescentes por seis anos, e constataram que um grande número de casos inicialmente diagnosticado como periodontite juvenil localizada evolui para periodontite juvenil generalizada.

A importância do diagnóstico precoce, avaliando crista óssea em radiografias de crianças, deve-se ao fato de que, dentre as periodontites de estabelecimento precoce, tem-se, em diferentes idades, diferentes estágios de uma mesma doença, que se estabeleceu, provavelmente, na infância e que não foi adequadamente e em tempo diagnosticada ${ }^{24}$. É possível realizar comparações de resultados de dados de prevalência de periodontite na dentição decídua e na permanente e concluir-se que o início da destruição periodontal em adultos pode ser descoberta na infância ${ }^{25}$. COGEN et al (1992) em um estudo retrospectivo e transversal sugerem que uma ampla porcentagem dos pacientes com periodontite juvenil que possuíam radiografias das dentiçōes mista e decídua apresentavam evidências de envolvimento periodontal muitos anos antes do início da puberdade. Uma das implicaçoos deste fato seria a possibilidade de que a periodontite juvenil frequentemente comece nos anos prépuberais, na dentição decídua. Isto indica a necessidade de uma avaliação periodontal atenta em todas as crianças, incluindo a determinaçao rotineira de sondagem. peridontal. Provavelmente, a única forma de ter-se informação sobre a idade de estabelecimento da destruição periodonta? seja através de pesquisa epidemiológica e de estudos clínicos longitudinais ${ }^{25}$.

VAN DER VELDEN et al (1989 consideraram que a doença destrutiva er jovens adolescentes pareceu ser associada corr. placa e inflamação gengival. Em outro estudc CARLOS et al (1988) constatam que : chance de que a perda de inserção se:

*Trabalho vencedor do $8^{\circ}$ Prêmio Estímulo Kolynos.

**Acadêmica da Faculdade de Odontologia; Bolsista do Programa Especial de Treinamento.

***Professor Assistente das Disciplinas Periodontia I e II da UFRGS e da ULBRA; Doutor em Periodontia.

R. José do Patrocínio, 655/13 - Cidade Baixa - CEP: 90050-003 - Porto Alegre - RS.

Av. Venâncio Aires, 449/904 - Bom Fim - CEP: 90040-193 - Porto Alegre - RS. 
diagnosticada em um dado sítio é aumentada em cinco vezes quando há cálculo presente, 16,5 vezes na presença de ambos cálculo e sangramento gengival, e em torno de 37 vezes quando todas as três variáveis de exposição estão presentes, ou seja, cálculo, sangramento e microbiota. Por outro lado. em um estudo de 1993, ALBANDAR infere que não há diferenças entre os adolescentes com periodontite juvenil e os sem periodontite juvenil na extensão da inflamaçāo gengival, ou na quantidade de acúmulo de placa dental e depósitos de cálculo sobre os dentes.

Nas formas mais avançadas da doença periodontal, a perda de inserção do tecido conjuntivo e a migraçāo apical do epitélio dento-gengival são achados importantes. Com a finalidade de avaliar a quantidade de tecido perdido na doença periodontal e também identificar a extensão apical da lesão inflamatória, devem ser registrados os seguintes parâmetros: a) profundiade de sondagem; b) nível de inserção (sondagem do nível de inserção); c) envolvimento de furca; e d) mobilidade dentária ${ }^{11}$.

Estudos epidemiológicos mostram que a prevalência de periodontite juvenil varia desde $0.1 \%^{19.20}, 0.112 \%^{13}, 0.15 \%^{16}$, de 0.1 a $0.2 \%^{26}, 0.32 \%^{18}, 1.8 \%^{2}$, sendo esta diagnosticada algumas vezes somente através de parâmetros clínicos de mensuração e outras aliando estes com radiográficos.

Esta larga variação nos estudos epidemiológicos de prevalência possivelmente pode ser explicada pelos diferentes critérios usados para diagnosticar periodontite juvenil, ou pelo estudo de populações com diferentes margens de idade ${ }^{13,18}$.

Alguns estudos apresentam a prevalência de perda de inserção periodontal igualmente diagnosticada somente clinicamente ou aliando estes exames com o radiográfico, sendo que constatam que radiograficamente a perda de inserção periodontal parece ser menor ${ }^{2.20}$. VAN DER VELDEN et al em estudo de 1989 encontrou uma prevalência de perda de inserção de $5 \%$ em um grupo de adolescentes com uma média de 15 anos de idade.

Em vista disso, o objetivo do presente estudo foi verificar a prevalência de perda de inserção periodontal em adolescentes de 12 a 16 anos de idade em uma escola pública de Porto Alegre.

\section{MATERLAIS E MÉTODOS \\ AMOSTRA}

O trabalho foi realizado na Escola Estadual de $1^{\circ}$ e $2^{\circ}$ Graus Álvaro Alves da Silva Braga. De 120 alunos matriculados no turno da tarde, 64 com idades de 12 a 16 anos participaram do estudo mediante a assinatura de um consentimento informado por seus responsáveis.

\section{PROCEDIMENTOS DE COLETA} DE DADOS

A presença de perda de inserção periodontal (PI) foi verificada nos ângulos mésio-vestibulares (MV), disto-vestibulares (DV), e no centro das faces vestibulares (V) e linguais/palatinas (L/P) dos primeiros molares permanentes e incisivos permanentes. Os dentes ausentes ou que se apresentavam como restos radiculares foram desconsiderados no exame periodontal.

A PI foi mensurada, quando presente, pelo uso de uma sonda periodontal milimetrada tipo Willians (Neomar, SP). A mensuração foi sempre feita por apenas um examinador treinado, dispondo de espelho bucal e iluminação padrão, procurando usar uma força de trabalho de não mais que $25 \mathrm{~g}$. $O$ treinamento para isto pode ser feito inserindo-se gentilmente a ponta da sonda sob a unha sem causar dor ou desconforto'.

$O$ nível de inserção periodontal foi definido como a distância em milímetros do limite amelo-cementário à porção mais apical sondável, próxima ao fundo do sulco/bolsa. Para cada sítio duas medidas foram feitas, a distância da margem gengival ao limite amelo-cementário, e a distância da margem gengival ao fundo do sulco/bolsa. O nível de inserção para um dado sítio foi, então, calculado subtraindo-se o primeiro do segundo ou, no caso de recessão gengival, somando-se os dois ${ }^{4}$. Foi considerado PI quando esta mensuração excedeu de 2 a 3 $\mathrm{mm}^{14}$.

As mensurações foram anotadas em ficha clínica própria para cada indivíduo, bem como a presença de sangramento à sondagem em todos os locais examinados.

Os indivíduos diagnosticados como tendo perda de inserção periodontal no estudo foram convidados a participar voluntariamente de investigação adicional e tratamento.

\section{PROCEDIMENTOS DE ANÁLISE} DOS DADOS

Os dados obtidos das mensurações das perdas de inserçāo periodontal foram tabulados e apresentados através de valores absolutos e porcentuais.

\section{RESULTADOS}

De 120 alunos matriculados no turno da tarde na escola, 64 alunos aceitaram participar voluntariamente do estudo. $\mathrm{O}$ grupo de participantes ficou subdividido em 18 indivíduos com 12 anos, 12 com 13 anos, 14 com 14 anos, 6 de 15 anos e 14 de 16 anos. Assim, obteve-se um total de 3048 sítios examinados, sendo que nos grupos dos 13 aos 16 anos encontravam-se indivíduos com ausência de um dos primeiros molares permanentes ou sua total destruição coronária por cárie, estes foram desconsiderados no exame periodontal (TAB. I).

TABELA 1. Distribuiçāo dos indivíduos examinados segundo idade e número total de sitios periodontais examinados, Porto Alegre, 1998.

\begin{tabular}{|c|c|c|}
\hline Idade & $\begin{array}{c}\mathbf{N}^{\circ} \text { de } \\
\text { Pacientes }\end{array}$ & $\begin{array}{c}\mathbf{N}^{0} \text { total } \\
\text { de sítios examinados }\end{array}$ \\
\hline 12 anos & 18 & 864 \\
\hline 13 anos* $^{*}$ & 12 & 572 \\
\hline 14 anos $^{*}$ & 14 & 668 \\
\hline 15 anos* $^{*}$ & 6 & 284 \\
\hline 16 anos $^{* *}$ & 14 & 660 \\
\hline TOTAL $^{\prime}$ & 64 & 3048 \\
\hline
\end{tabular}

* grupo contendo I paciente com perda de $1^{\circ}$ molar permanente por cárie.

** grupo contendo 3 pacientes com perda de $1^{\circ}$ molar permanente por cárie.

Constatou-se que dos 3048 sítios examinados, $147(4.82 \%)$ apresentaram PI, enquanto que $276(9.05 \%)$ sangramento independentemente da presença de PI. No grupo com 12 anos de idade, verificou-se a menor prevalência de PI (TAB. 2).

TABELA 2. Distribuição das idades dos pacientes segundo o total de sítios periodontais examinados, com perda de inserção (PI) e com sangramento, Porto Alegre, 1998.

\begin{tabular}{|c|c|c|c|c|c|}
\hline \multirow{2}{*}{ Idade } & $\begin{array}{c}\text { No }^{\circ} \text { total } \\
\text { de faces } \\
\text { exami- } \\
\end{array}$ & \multicolumn{2}{|c|}{\begin{tabular}{c}
\multicolumn{2}{|c|}{ Sítios } \\
com PI
\end{tabular}} & \multicolumn{2}{c|}{$\begin{array}{c}\text { Faces dentais } \\
\text { com } \\
\text { nangramento }\end{array}$} \\
\cline { 3 - 7 } & $\mathbf{n}$ & $\%$ & $\mathbf{n}$ & $\%$ \\
\hline 12 anos & 864 & 26 & 3.00 & 66 & 7.63 \\
\hline 13 anos & 572 & 30 & 5.24 & 82 & 14.33 \\
\hline 14 anos & 668 & 24 & 3.59 & 50 & 7.48 \\
\hline 15 anos & 284 & 24 & 8.45 & 45 & 15.84 \\
\hline 16 anos & 660 & 43 & 6.51 & 33 & 5.00 \\
\hline TOTAL & 3048 & 147 & 4.82 & 276 & 9.05 \\
\hline
\end{tabular}

Observou-se que o grupo com 12 anos mostrou-se com a maior quantidade de indivíduos com nenhuma perda de inserção. Com o aumento da idade dos indivíduos constatou-se uma menor quantidade de indivíduos com esta característica. Dos indivíduos com PI em até dois sítios e naqueles em mais de dois sítios não observa-se um padrão de distribuição (TAB. 3).

Dos sítios periodontais incluídos no exame que apresentaram PI, o que apresentou a maior prevalência foi a face vestibular com 99 faces sendo que houve maior ocorrência de PI sem sangramento (79.79\%). Nos outros sítios houve prevalência de PI com sangramento. No entanto, considerando-se o valor total de PI (147), encontra-se uma maior prevalência de PI sem sangramento (61.22\%) (TAB. 4). 
Analisando-se estes valores em relação ao número total de sítios estudados, obtem-se uma prevalência de $1.87 \%$ de PI com sangramento e de $2.95 \%$ de PI sem sangramento (TAB.5).

TABELA 3. Distribuição dos pacientes por idade, ausência de perda de inserção (PI), presença de até dois sitios periodontais com PIe com mais de dois com PI, Porto Alegre, 1998.

\begin{tabular}{|c|c|c|c|c|c|c|c|}
\hline \multirow{2}{*}{ Idade } & \multirow{2}{*}{$\begin{array}{c}N^{*} \text { de } \\
\text { Pacient- } \\
\text { tes }\end{array}$} & $\begin{array}{c}\text { Sem perda de } \\
\text { inserçá }\end{array}$ & \multicolumn{2}{|c|}{$\begin{array}{c}\text { Com até dois } \\
\text { sítios com PI }\end{array}$} & \multicolumn{2}{c|}{$\begin{array}{c}\text { Com mais de } \\
\text { dois sítios } \\
\text { com PI }\end{array}$} \\
\cline { 3 - 8 } & $\mathrm{n}$ & $\%$ & $\mathrm{n}$ & $\%$ & $\mathrm{n}$ & $\%$ \\
\hline 12 anos & 18 & 8 & 44.44 & 5 & 27.77 & 5 & 27.77 \\
\hline 13 anos & 12 & 4 & 33.33 & 4 & 33.33 & 4 & 33.33 \\
\hline 14 anos & 14 & 3 & 21.42 & 7 & 50.00 & 4 & 28.57 \\
\hline 15 anos & 6 & 1 & 16.66 & 4 & 66.66 & 1 & 16.66 \\
\hline 16 anos & 14 & 2 & 14.28 & 3 & 21.42 & 9 & 64.28 \\
\hline TOTAL & 64 & 18 & 28.12 & 23 & 35.93 & 23 & 35.93 \\
\hline
\end{tabular}

TABELA 4. Distribuição dos sítios periodontais examinados segundo a perda de inserção (PI) total, PI com sangramento e PI sem sangramento, Porto Alegre, 1998.

\begin{tabular}{|c|c|c|c|c|c|}
\hline \multirow{2}{*}{ Sitios } & \multirow{2}{*}{$\begin{array}{c}\text { Monta- } \\
\text { nte com } \\
\text { PI }\end{array}$} & \multicolumn{2}{|c|}{ PI com } & \multicolumn{2}{|c|}{ PI sem } \\
\cline { 3 - 6 } & $\mathrm{n}$ & $\%$ & $\mathrm{n}$ & $\%$ \\
\hline $\begin{array}{c}\text { Mésio- } \\
\text { vestibular }\end{array}$ & 13 & 11 & 84,61 & 2 & 15,38 \\
\hline $\begin{array}{c}\text { Disto- } \\
\text { vestibular }\end{array}$ & 8 & 8 & 100 & $\mathrm{Z}$ & - \\
\hline Vestibular & 99 & 20 & 20,20 & 79 & 79,79 \\
\hline $\begin{array}{c}\text { Lingual } \\
\text { palatina }\end{array}$ & 27 & 18 & 66,66 & 9 & 33,33 \\
\hline TOTAL & 147 & 57 & 38.77 & 90 & 61.22 \\
\hline
\end{tabular}

TABELA 5. Distribuição dos grupos por idade segundo faces examinadas, sitios com perda de inserçäo (PI) total, PI com sangramento e PI sem sangramento, Porto Alegre, 1998.

\begin{tabular}{|c|c|c|c|c|c|c|c|}
\hline \multirow{2}{*}{ Idade } & \multirow{2}{*}{$\begin{array}{l}\text { No total } \\
\text { de faces } \\
\text { exami- } \\
\text { nadas }\end{array}$} & \multicolumn{2}{|c|}{ PI total } & \multicolumn{2}{|c|}{$\begin{array}{l}\text { PI com } \\
\text { sangra- } \\
\text { nento }\end{array}$} & \multicolumn{2}{|c|}{$\begin{array}{l}\text { PI sem } \\
\text { sangra- } \\
\text { mento }\end{array}$} \\
\cline { 5 - 9 } & $\mathrm{n}$ & $\%$ & $\mathrm{n}$ & $\%$ & $\mathrm{n}$ & $\%$ \\
\hline 12 anos & 864 & 26 & 3.00 & 12 & 1.38 & 14 & 1.62 \\
\hline 13 anos & 572 & 30 & 5.24 & 15 & 2.62 & 15 & 2.62 \\
\hline 14 anos & 668 & 24 & 3.59 & 3 & 0.44 & 21 & 3.14 \\
\hline 15 anos & 284 & 24 & 8.45 & 19 & 6.69 & 5 & 1.76 \\
\hline 16 anos & 660 & 43 & 6.51 & 8 & 1.21 & 35 & 5.30 \\
\hline TOTAL & 3048 & 147 & 4.82 & 57 & 1.87 & 90 & 2.95 \\
\hline
\end{tabular}

Dos sítios estudados, no mésio-vestibular obteve-se a maior quantidade de sangramento à sondagem sem perda de inserção, ou seja, 74 sítios, que corresponde a 9,71\% ('TAB. 6).

TABELA 6. Distribuição dos sitios periodontais examinados segundo a presença de sangramento à sondagem sem perda de inserção,
Porto Alegre, 1998.

\begin{tabular}{|c|c|c|c|}
\hline Sítio & $\begin{array}{c}\text { No de } \\
\text { sítios } \\
\text { exami- } \\
\text { nados }\end{array}$ & $\begin{array}{c}\text { Sangramento } \\
\text { sem perda de } \\
\text { inserção }\end{array}$ \\
\cline { 3 - 4 } & $\mathrm{n}$ & $\%$ \\
\hline Mésio-vestibular & 762 & 74 & 9.71 \\
\hline Disto-vestibular & 762 & 56 & 7.34 \\
\hline Vestibular & 762 & 55 & 7.21 \\
\hline Lingual/palatina & 762 & 33 & 4.33 \\
\hline TOTAL & 3048 & $\mathbf{2 1 8}$ & 7.15 \\
\hline
\end{tabular}

\section{DISCUSSÃO}

Este estudo teve como objetivo verificar a prevalência de perda de inserção periodontal em um grupo de adolescentes de 12 a 16 anos de idade. De 3048 sítios examinados, 147 (4.82\%) apresentaram perda de inserção periodontal. Este achado apresenta-se relativamente alto quando comparado aos trabalhos de VAN DER VELDEN et al (1989) e de LÖE e BROWN (1991), que encontraram os respectivos valores $2.3 \%{ }^{26}$ e $2.17 \%^{16}$. Por outro lado, também encontra-se na literatura resultados tais como o de CARLOS et al em um trabalho de 1988, no qual há uma prevalência de perda de inserção de $16.9 \%^{8}$. Entretanto, os dados estão em concordância com os resultados de VAN DER VELDEN et al (1989) para adolescentes com média de idade de 15 anos, para os quais encontrou-se uma prevalência de $5 \%$ de perda de inserção periodontal. Esta diferença de dados encontrada pode ser explicada pelos diferentes critérios de avaliação empregados, ou por utilizarem grupos populacionais com diferentes margens de idade ${ }^{13,18}$. Um dos critérios que varia enormemente entre os estudos é a partir de que valor é considerado como perda de inserção periodontal. Na presente investigação os valores de 2-3 $\mathrm{mm}$ foram estabelecidos em concordância com diversos estudos histológicos e clínicos de periodontos sadios ${ }^{8.14 .15,23}$.

Alguns estudos sobre prevalência de recessão gengival apresentam dados que variam desde 3 a $100 \%{ }^{17}$, dependendo da idade do grupo populacional estudado. Entretanto, considerando-se dados referentes a populaçōes jovens, estes valores ficam em torno de $8.95 \%{ }^{5}$ $30 \%$ e $60 \%$, este último valor refere-se exclusivamente à face vestibular ${ }^{17}$. No presente estudo, é pertinente considerar que a perda de inserção sem sangramento encontrada (2.95\%) seja recessão gengival, a qual foi mais prevalente nas faces vestibulares. Uma vez que o sangramento à sondagem no fundo do sulco/ bolsa gengival está associado com a presença de um infiltrado de células inflamatónias nesta área, a ocorrência deste sangramento seria um sinal importante de atividade de doença periodontal ${ }^{14}$. A causa para a recessão gengiva para alguns autores é atribuída ao trauma proveniente de irritantes mecânicos bacterianos, ou mesmo fatores clínicos predominando um ou mais destes em determinado sítio e ser difícil a identificação ${ }^{212}$

$\mathrm{O}$ presente estudo avaliou a perda de inserção periodontal nos incisivos e primeiros molares permanentes, baseando-se em estudo: que mostram que a periodontite $d$ estabelecimento precoce inicia-se de form localizada nestes dentes, os quais erupcionar: na mesma época na cavidade bucal transformando-se em uma doença generalizada se não tratada $4,6,7,11,25$.

Levando-se em consideração que o: hábitos de higiene bucal para a maioria da pessoas não inclui o uso rotineiro de instrumento de limpeza da região interproximal ${ }^{10}$, como fi: dental e escova interdental, sabe-se que o estad inflamatónio de sítios periodontais em superficie livres seja diferente de sítios em superfície proximais ${ }^{910}$. Portanto, a presente investigaça:: periodontal abrangeu dois sítios em superfície livres e dois em superfícies proximais encontrando uma prevalência de PI cor sangramento em regióes proximais maior do quisem sangramento.

Em função dos altos valores encontrado: pela presente investigação e pelos dado encontrados na literatura, é pertinente suger: que todo o cirurgiāo-dentista insira o exam. periodontal como rotina em sua prática clínica

\section{CONCLUSÄO}

Sob as condiçōes do estudo, é possive concluir que:

$>$ A prevalência de perda de inserçä: periodontal foi de $4.82 \%$.

$>$ A prevalência de perda de inserçá: periodontal com sangramento foi $d$. $36.77 \%$ e sem sangramento $\dot{c}$ : $61.22 \%$.

$>$ A face vestibular mostrou-se com : maior prevalência de perda de inserça:

\section{ABSTRACT}

The aim of this study was to verify the prevalence of loss of attachment in 12-16 yeaold adolescents of a public school in Porto Aleg:The prevalence of loss of attachment wz determined by means of periodontal probing $a$ mesial, distal, buccal and lingual surfaces $a$ incisors and first permanent molars. A traine: examiner performed the examination with: Williams periodontal probe, odontoscope astandard illumination. The level of attachme was defined as the distance in millimeters from the cementum-enamel junction to the mos apically probable portion, close to the bottcm of the sulcus/pocket. For each site, $w$ measurements were performed, the distan from the gingival margin to the cementur. enamel junction, and the distance from the gingival margin to the bottom of the sulcess pocket. The level of attachment for a site nz calculated subtracting the first and the secom: 
or, in case of gingival recession, adding both. Loss of attachment was considered when this measure exceeded $2 \mathrm{~mm}$ in buccal-lingual surfaces and $3 \mathrm{~mm}$ in proximal surfaces. 3048 sites were examined, showing a prevalence of $4.82 \%$ of loss of attachment. From these, $36.77 \%$ bled on probing and $61.22 \%$ did not. The buccal surface was the one with the highest prevalence of loss of attachment.

\section{KEYWORDS:}

periodontal attachment;epidemiology; adolescents.

\section{REFERÊNCIAS BIBLIOGRÁFICAS}

I.AINAMO, J.; BARMES, D.; BEAGRIE, G. et al. Development of the World Health Organization (WHO) Community Periodontal Index of Treatment Needs (CPITN). Int. Dental J. London, v.32, n.3, p.281-291, Sept. 1982.

2.ALBANDAR, J. M. Juvenile Periodontitis: Pattern of Progression and Relationship to Clinical Periodontal Parameters. Comminity Dent. Oral Epidemiol., Copenhagen, v.21, p.185-189, Aug. 1993.

3.ALBANDAR, J. M.; BROWN, L. J.; BRUNELLE, J. A. et al. Gingival State and Dental Calculus in Early-Onset Periodontitis. J. Periodontol., Chicago, v.67, n. 10, p.953-959, Oct. 1996.

4.ALBANDAR, J. M.; BROWN, L. J.; LÖE, H. Dental Caries and Tooth Loss in Adolescents with EarlyOnset Periodontitis.J. Periodontol., Chicago, v67, n. 10. p.960-967, Oct. 1996.

5.AZEVEDO, M. P.; SUSIN, C.; RÖSING, C. K. Prevalência de Recessão Gengival em Escolares e sua Relaçăo com Estado Inflamatório. In: REUNIÁO ANUAL DA SOCIEDADE BRASILEIRA DE PESQUISA ODONTOLÓGICA, 14., 1997, Águas de São Pedro. São Paulo, Águas de São Pedro: Sociedade Brasileira de Pesquisa Odontológica, 1997. p. I37.

6.BAER, P. N. The Case for Periodontosis as a Clinical Entity. J. Periodontol., Chicago, v.42, n.8, p.516520, Aug. 1971

7.BROWN, L. J.; ALBANDAR, J. M.; BRUNELLE, J. A. et al. Early-onset Periodontitis: progression of attachment loss during 6 years. J. Periodontol., Chicago, v.67, n.10, p.968-975, Oct. 1996.

8.CARLOS, J. P.; WOLFE, M. D.; ZAMBON, J.J.; et al Periodontal Disease in Adolescents: some clinical and microbiologic correlates of attachment loss. J. Dent. Res., Chicago, v. 7, n.12, p.1510-1514, Dec. 1988

9.CATON, I.; BOUWSMA, O.; POLSON, A et al. Effects of Personal oral Hygiene and Subgingival Scaling on Bleeding Interdental Gingiva. I. Periadontol., Chicago, v.60, n.2, p.84-90, Feb. 1989.

10.CATON, J.; BLIEDEN, T. M.; LOWENGUTH, R A. et al. Comparison Between Mechanical Cleaning and an Antimicrobial Rinse for the Treatment and Prevention of Interdental Gingivitis. J. Clin. Periodontol., Copenhagen, v.20, n.3, p.172178, Mar. 1993

11.COGEN, R. B.; WRIGHT, J. T.; TATE, A. L. Destructive Periodontal Disease in Healthy
Children. J. Periodontol., Chicago, v.63, n.9, p.761.765, Sept. 1992.

12.HANSEN, B. F; GJERMO P; BERGWITZ-LARSEN K. R. Periodontal Bone Loss in 15-year-old Norwegians. J. Clin. Pcriodontol., Copenhagen, v.11, n.2, p.125.131, Feb. 1984.

13.KRONAUER, E.; BORSA, G.; LANG, N. P. Prevalence of Incipiente Juvenile Periodontitis at Age 16 years in Switzerland. J. Clin. Periodontol. Copenhagen, v.13, n.2, p.103-108, Feb. 1986.

14.LINDHE, J. Tratado de Periodontologia Clínica. 2.ed. Rio de Janeiro: Guanabara Koogan, 1992. 493p.

15.LISTGARTEN, M. A. Periodontal Probing: what does it mean? J. Clin. Periodontol., Copenhagen, v. 7, n.3, p.165-176, June 1980 .

16.LÖE, H. AND BROWN, J. Early Onset Periodontitis in the United States of America. J. Periodontol., Chicago, v.62, n. 10, p.608-616, Oct. 1991.

17.LÖE, H.; ANERUD, A; BOYSEN, H. The Natural History of Periodontal Disease in Man: prevalence, severity, and extent of gingival recession. J. Periodontol., Chicago, v. 63, n.6, p.489-495, June 1992.

18.LÓPEZ, N. J.; RIOS, V.; FERNÁNDEZ, O. Prevalence of Juvenile Periodontitis in Chile. J. Clin. Periodontol., Copenhagen, v.18, n.7, p.529.533, Aug. 1991.

19.SAXBY, M. Prevalence of Juvenile Periodontitis in a British School Population. Community Dent. Oral Epidemiol., Copenhagen, v.12, n.3, p.185-187, June 1984.

20.SAXÉN, L. Prevalence of Juvenile Periodontitis in Finland. J. Clin. Periodontol., Copenhagen, v.7, n.3, p.177-186, June 1980

21.SMITH, R. G. Gingival Recession: reappraisal of an enigmatic condition and a new index for monitoring. J. Clin. Periodontol., Copenhagen, v.24, n.3, p.201-205, Mar. 1997.

22.SMUKLER, H.; LANDSBERG, J. The Toothbrush and Gingival Traumatic Injury. J. Periodontol. Chicago, v.55, n.12, p.713-719, Dec. 1984.

23.TEN CATE, A. R. Histologia Bucal: desenvolvinento, estrutura c funcão. 2.ed. Rio de Janeiro: Guanabara Koogan, 1988. 395p.

24.TOLEDO, B. E. C.; RÖSING, C. K.;IARRIM, N. C et al. Análise Radiográfica da Reabsorçāo da Crista Óssea em Crianças de 6 a 12 anos. Stomatos, Canoas, n.4, p.29-33, I. Sem. 1997.

25.VAN DER VELDEN, U. The Onset Age of Periodontal Destruction. J. Clin. Periodontol., Copenhagen v.18, n.6, p.380-383, July 1991 .

26. VAN DER VELDEN, U.; ABBAS, F; VAN STEENBERGEN, T. J. M. et al. Prevalence of Periodontal Breakdown in Adolescents and Prevalence of Actinobacillus actinomyectemcomitans in Subjects with Attachment Loss. J. Periodontol. Chicago, v.60, n. I 1, p.604-610, Nov. 1989. 